\title{
Cognitive Interference Management Techniques for the Spectral Co-existence of GSO and NGSO Satellites
}

\author{
Ameneh Pourmoghadas, Shree Krishna Sharma, Symeon Chatzinotas, \\ Björn Ottersten \\ SnT - securityandtrust.lu, University of Luxembourg, \\ ameneh. pourmoghaddaslangroudi@uni.lu
}

\begin{abstract}
One of the main challenges in the co-existence of geostationary satellite orbit (GSO) and non-geostationary satellite orbit (NGSO) satellite networks is to mitigate the in-line interference caused by an NGSO satellite to the GSO earth terminal, while the NGSO satellite is crossing the GSO satellite's illumination zone. The method recommended in ITU-R S.1325-3 involves utilizing a range-based power control on the NGSO satellite for downlink communication to the NGSO earth terminals. In this paper, we investigate a cognitive range-based power control algorithm while taking into account the imposed interference level to the GSO fixed satellite service (FSS) system. Results show that the proposed cognitive power control algorithm can mitigate the harmful in-line interference on the GSO terminal receiver, while also providing the desired link quality for the NGSO system. More importantly, we formulate and solve an optimization problem with the objective of minimizing the intersite distance (ISD) of the GSO-NGSO earth user-terminals. Finally, we develop an analytical method to calculate the ISD between GSO and NGSO earth terminals and validate this with the help of simulation results.
\end{abstract}

\section{Introduction}

Satellites communications today provide many possibilities and opportunities to achieve better quality of service and extend backhaul services to anywhere and any-time [1]. However, fixed satellite service (FSS) involves deployment of a large number of user terminals on the earth. Further study on uncoordinated techniques for satellite systems is required to mitigate the interference caused by an adjacent satellite network to FSS systems. In general, the main contribution of the networking solutions for satellite networks are involved in techniques for satellite energy saving [2], efficient resource allocation [3], and FSS system interference mitigation $[1,4]$. Studies on efficient usage of satellite resources and their co-existence with either terrestrial systems or other satellite networks exist in the literature $[5,6]$. The co-existence of satellite systems with either other satellite systems or terrestrial networks can be achieved by employing suitable resource allocation and interference mitigation techniques [7], [8]. Apart from 
the requirement of finding intelligent (or cognitive) resource allocation, it is important to ensure that the co-existence of multi-satellite systems does not cause interference to other co-channel systems such as FSS systems, mobile satellite services (MSS), or terrestrial systems. In particular, the interference mitigation is more complicated considering a practical dual satellite system (DSS) scenario, in which two satellites (e.g., GSO-NGSO satellites) operate over the same coverage area while sharing the spectrum bands [9]. In the co-existence scenarios of GSONGSO networks, in-line interference arises whenever an NGSO satellite passes through a line of sight path between an earth station and a GSO satellite [10]. It occurs due to the fact that an NGSO satellite may create/receive interference through its sidelobe or mainlobe to the GSO system (please see Fig. 1). To understand this scenario better, we can consider the co-existence of O3b satellites within $\pm 5^{\circ}$ latitude from the equator and GSO Eutelsat KA-SAT satellite [longitude: $9.0^{\circ}$, inclination: $0.01^{\circ}$ ]. The O3b satellite which uses the medium earth orbit (MEO) constellation and shares the frequency 18.8-19.3 GHz in downlink communication with the GSO system, has a high potential to cause interference to the GSO FSS system. In this context, one of the key challenges that has been identified in ITU Radio Regulations and European Space Agency is the need to explore efficient techniques to mitigate the in-line interference for the spectral co-existence of GSO-NGSO satellite networks.

\subsection{Related Literature}

Various studies have been conducted to address challenges in interference mitigation in the literature and ITU-R reports [5]- [10]. Concerning the uncoordinated (cognitive) techniques some of these approaches are: satellite selection strategies with the largest angular discrimination [11]; spot turnoff method [12]- [13]; and power control technique [10]. In the last technique, a cognitive transmission power algorithm is used to provide the required signal to noise ratio (SNR) at the receiver and maintain the interference level at the victim receiver. Most of the studies on dynamic transmission power controls on satellite stations involves on-board energy saving, without attention to investigations on controlling the imposed interferences on the victim receiver $[17,18]$. Besides, majority of the research works in the literature have been carried out for individual satellite systems. Wherein, the integration between different satellite constellations plays a key role in moving towards the next generation of the satellite networks. A more related work to the objective of current paper can be found in [19], [10]. Authors in [19] find the minimum separation distance between terrestrial base station (BS) and FSS earth station by considering the interference caused from terrestrial BS to the FSS earth station. In [10] a cognitive transmission power algorithm is used to provide the required signal to noise ratio (SNR) at the receiver and maintain the interference level at the victim receiver. Nevertheless, the impact of the inter-site distance (ISD) between GSO-NGSO earth terminals on the interference level of the victim GSO earth receiver is not studied. 


\subsection{Motivation and Contributions}

According to the ITU-R S.1325-3 recommendation, adaptive power control on range in the downlink is one of the useful interference mitigation techniques in facilitating spectrum sharing between GSO and NGSO networks. This technique is left for further investigation in ITU-R S.1325-3. Besides, studies on earth terminal deployment strategies (such as inter-site distance among terminals) is one of the possible approaches for the efficient use of limited spectrum bands and network's capacity improvement [19]. In this context, the main contributions of this paper are as follows: 1) We utilize the cognitive power control in [10] based on the range (distance between NGSO satellite and NGSO earth terminal) in the co-existence scenario of GSO and NGSO FSS systems. 2) We analyse the feasibility of the cognitive range-based power control method in terms of GSO-NGSO geometry. 3) More importantly, we develop a theoretical model for minimum possible between the GSO and NGSO FSS earth terminals through analysis. 4) We present the improvements on the minimum ISD between FSS earth terminals using the proposed model, in which the NGSO satellite does not utilize any dynamic power control method on-board.

The rest of the paper is as follows: Section 2 provides the system model and the in-line interference scenario. The cognitive range-based power control is defined in Section 3. We propose a method to determine the minimum ISD between FSS earth terminals in Section 4 . Section 5 provides the analysis and simulation results. Finally, we draw the conclusion in Section 6 .

\section{System Model And Interference Scenario}

In accordance with the co-existence of GSO-NGSO FSS systems while sharing spectrum on downlink ${ }^{1}$ and uplink, the following in-line interference scenarios are probable to occur [10]: 1) Interference from the NGSO satellite to the GSO earth terminal in the downlink. 2) Interference from the GSO earth terminal to the NGSO satellite in the uplink. 3) Interference from the GSO satellite to the NGSO earth terminal in the downlink. 4) Interference from the NGSO earth terminal to the GSO satellite in the uplink. In this paper, we consider the GEO and MEO constellations, where an O3b satellite in MEO orbit is chosen as the use case scenario causing harmful in-line interference to the GSO earth terminal. Earth terminals are the user terminals operating in Ka-band in the FSS system such as VSAT. Reports in ITU-R S.1325-3 indicate that the number of interference events from NGSO satellite on GSO earth terminals observed more in downlink communication. Therefore, in this work, we focus on the downlink in-line interference from NGSO satellite to the GSO earth terminal (item (1) from the above listed bullets, please see Fig. 1), and we leave the rest of the scenarios for future work. Throughout this paper, we have assumed that the transmitters of the NGSO satellite communicate with their wanted receivers (NGSO earth terminal) along the boresight direction (i.e., off-axis angle $=0^{\circ}$ );

\footnotetext{
${ }^{1}$ Throughout this article, the interference in downlink refers to the user-link interfer-
} ence. 


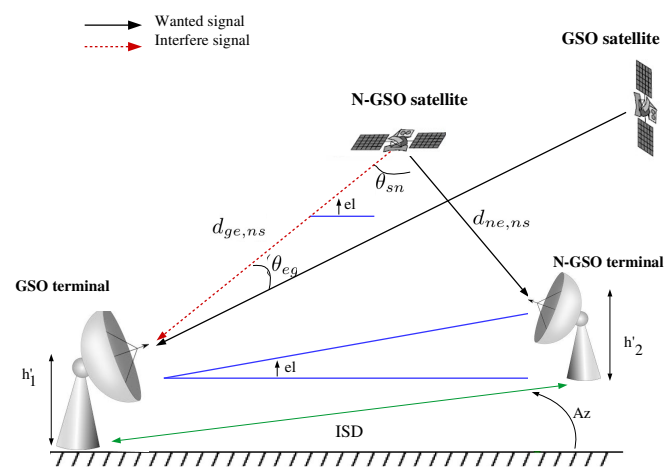

Fig. 1: The interference scenario between GSO and NGSO FSS systems.

which means that the antenna gain of the NGSO satellite and the NGSO earth terminal remains fixed in the downlink communication. In our system model, we have assumed free space loss (FSL) on the direct or interference channels, and fading phenomena such as diffraction or tropospheric propagation effects etc., are not taken into account.

\section{Cognitive and Range-Based Power Control Algorithms}

According to the ITU-R S.1325-3 recommendation, NGSO satellite can decrease or increase the transmission power based on the range, while providing the desired SNR level to the NGSO earth terminal. This is known as a range-based power control method. To enhance this method, we propose a cognitive rangebased power control algorithm at the NGSO satellite. In the proposed algorithm, the NGSO satellite will decrease or increase its transmission power while its distance to the NGSO earth terminal is getting shorter or longer accordingly. We have considered two threshold values for optimizing the transmission power of the NGSO satellite. The minimum SNR level at the wanted receiver, $S N R_{\text {min }}$, and the tolerable interference threshold at the victim receiver, $I_{t h}$. We define two power control methods in the following subsections. In the rest of this work, the following subscripts are used: $e$ for the earth system, $s$ for the satellite, $n$ for the NGSO system, and $g$ for the GSO system.

\subsection{Range-based Power Control Method}

To compute the transmission power on the NGSO satellite as a function of range, $d_{n e, n s}$ (see Fig. 1), we first need to find the SNR level at the NGSO earth terminal. Using the FSL model, the received power at the NGSO earth terminal from the NGSO satellite can be computed as:

$$
\operatorname{Pr} x_{n e}=\operatorname{Pt} x_{n s}\left(d_{n e, n s}\right) G t x_{n s}(0) G r x_{n e}(0) F S L\left(\lambda, d_{n e, n s}\right),
$$


where $\operatorname{Pr} x_{n e}[W]$ is the desired receive power at the input to the NGSO earth terminal antenna, $P t x_{n s}[W]$ is the transmit power of the NGSO satellite station, and function $F S L(\lambda, d)=\left(\frac{\lambda}{4 \pi d}\right)^{2}$, in which $\lambda$ is the wavelength. Gtx $x_{n s}(0)$, $G r x_{n e}(0)$ are the transmit and receive antenna gains of the NGSO satellite and earth terminal along the boresight direction, respectively. The received $S N R$ at the NGSO earth terminal is defined as:

$$
S N R=\frac{P t x_{n s}\left(d_{n e, n s}\right) G t x_{n s}(0) G r x_{n e}(0)}{N}\left(\frac{\lambda}{4 \pi d_{n e, n s}}\right)^{2},
$$

where $N[W]$ is the thermal noise power at the receiver and can be expressed as:

$$
N=k \times T_{n e} \times B W,
$$

where $k$ is the Boltzmann's constant $\left(1.38 \times 10^{-23}[\mathrm{~J} / \mathrm{K}]\right)$, and $T_{n e}[K]$ is the noise temperature at the NGSO earth terminal, and $B W[H z]$ is the receiver bandwidth. In order to guarantee $S N R_{\min }$ at the NGSO earth terminal the transmit power on the NGSO satellite as a function of range can be computed as follows.

$$
P t x_{n s}\left(d_{n e, n s}\right)=\frac{S N R_{\min } \times N}{G r x_{n e}(0) \times G t x_{n s}(0)}\left(\frac{4 \pi d_{n e, n s}}{\lambda}\right)^{2} .
$$

\subsection{Cognitive Range-based Power Control Method}

The range-based power control technique in Eq. (4) satisfies the required $S N R_{\text {min }}$ at the NGSO earth terminal receiver to close the link. To apply the range-based power control in the considered coexistence scenario, we re-write this technique by taking into account the interference threshold level $\left(I_{t h}\right)$ of the GSO earth terminal as following optimization problem ${ }^{2}$.

$$
\begin{array}{ll}
\operatorname{minimize} & \operatorname{Ptx}\left(d_{n e, n s}\right) \\
\text { C.1. } & S N R \geq S N R_{\min } \\
\text { C.2. } & I \leq I_{t h} \\
\text { C.3. } & P t x\left(d_{n e, n s}\right) \leq P t x_{\max },
\end{array}
$$

where $P t x_{\max }$ is the available maximum transmission power on NGSO satellite board. In Eq. (5), conditions $C .1$ is to take care of the NGSO FSS QoS requirement, $C .2$ is to avoid harmful interference on the GSO earth terminal, and C.3 is to make sure the range-based transmission power allocation does not exceed $P t x_{\max } . I$ is the interference ratio with respect to the wanted signal SNR at the GSO earth terminal as follows.

$$
I=\frac{\operatorname{Ptx}\left(d_{n e, n s}\right) G t x_{n s}\left(\theta_{n s}\right) G r x_{g e}\left(\theta_{g e}\right)}{F S L\left(\lambda, d_{g e, n s}\right)},
$$

\footnotetext{
${ }^{2}$ In this technique we have assumed that $I_{t h}$ level at the victim GSO earth termianl
} is known in public domain. 
where $\operatorname{Ptx}\left(d_{n e, n s}\right)[W]$ is the available transmit power at the NGSO satellite computed in Eq. (4), $d_{g e, n s}[m]$ is the distance between the GSO earth terminal and NGSO satellite, and $G t x_{n s}\left(\theta_{n s}\right), G r x_{g e}\left(\theta_{g e}\right)$ are the transmit and receive antenna gains at the off-axis angles of the NGSO satellite and GSO earth terminal respectively. The values of the parameters are given in the Table 1. Depending, on the geometry of the considered earth stations and satellites, it is obvious that the optimization problem in Eq. (5) may not be feasible when NGSO satellite is closer to the GSO earth station and far from the NGSO earth station. In this case, although the NGSO satellite can use the maximum power for its transmission to close the link with the NGSO earth station receiver, it may not satisfy the interference threshold level at the GSO earth station. Therefore, it is better to handover the transmission to the next NGSO satellite that has better link conditions. This challenge motivates us to find the possible minimum ISD between the GSO-NGSO earth terminals such that the threshold constraints (in Eq. (5)) are satisfied.

In following, we first discuss the geometry determination of ISD between earth stations; then we follow the optimization problem for computing the minimum distance, $I S D_{\min }$, between GSO and NGSO earth terminals.

\section{Proposed ISD Determination Method}

In this section, we develop an analytical method for finding the minimum ISD between the GSO and NGSO earth terminals by formulating an optimization problem. Our optimization problem will take into account the interference level from NGSO satellite to the GSO earth terminal in downlink communication. We have assumed that the position of GSO earth terminal is fixed, and we can only control the repositioning of the NGSO earth terminal. In this analysis it is assumed that satellite communication system operators have complete freedom in determining the geographical location of ground stations. First, let's find a relation between the ISD and the wanted range between NGSO satellite and earth terminal, $d_{n e, n s}$ (please see Fig. 2). Having the altitude, longitude and latitude of each earth terminal and satellite we can compute distance between any earth terminal to any given satellite $\left(d_{* e, * s}\right)$, as follows.

$$
d_{* e, * s}=r_{\text {sat }} \sqrt{1+\left(\frac{R_{E}^{2}}{r_{\text {sat }}}\right)^{2}-2\left(\frac{R_{E}^{2}}{r_{\text {sat }}}\right) \cos \omega \cos \Gamma},
$$

where $r_{\text {sat }}$ is the satellite radius, $R_{E}$ is the earth radius, $\omega$ is the difference in longitude, in degrees, between the earth station and the satellite, and $\Gamma$ is the earth station latitude in degrees. Let's denote local heights of the GSO and NGSO earth terminals as $h_{1}^{\prime}$ and $h_{2}^{\prime}$ respectively. Then, we have:

$$
L=\frac{h_{2}}{\tan \left(\varepsilon_{2}\right)}, h_{2}=d_{n e, n s} \times \sin \left(\varepsilon_{2}^{\prime}\right)+H, H=\left|h_{1}^{\prime}-h_{2}^{\prime}\right|,
$$

where $L$ is the horizontal distance of the NGSO satellite from the GSO earth terminal, $h_{1}$ and $h_{2}$ are the altitudes of the GSO and NGSO satellites from 


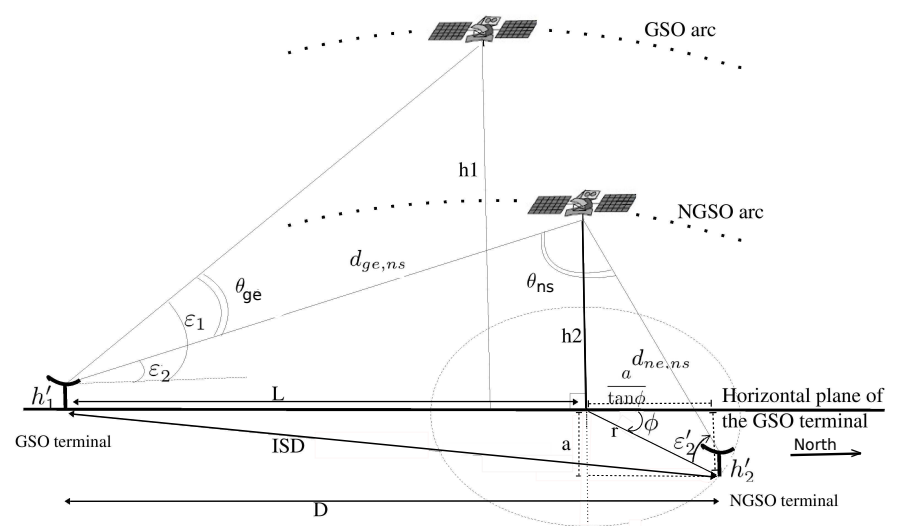

Fig. 2: Geometry of GSO and NGSO FSS systems with respect to the ISD.

the horizontal plane of the GSO terminal. $\varepsilon_{2}$ is the elevation angle of the NGSO satellite from the GSO earth terminal, and, $\varepsilon_{2}^{\prime}$ is the elevation angle of the NGSO satellite from the NGSO earth terminal. From Eq. (8), we can compute $L$ as:

$$
L=\frac{d_{n e, n s} \times \sin \left(\varepsilon_{2}^{\prime}\right)+H}{\tan \left(\varepsilon_{2}\right)} .
$$

Since $L$ is known, we can compute the horizontal distance between NGSO and GSO earth terminals, $D$, as follows.

$$
D=\frac{d_{n e, n s}+\sin \left(\varepsilon_{2}^{\prime}\right)+H}{\tan \left(\varepsilon_{2}\right)}+d_{n e, n s} \times \cos (\phi) \cos \left(\varepsilon_{2}^{\prime}\right) .
$$

Where $\phi$ is the azimuth angle of the NGSO earth terminal from the GSO earth horizon in degrees. ISD can be computed as

$$
I S D\left(\varepsilon_{2}, \varepsilon_{2}^{\prime}, \phi, d_{n e, n s}\right)=\sqrt{D^{2}+a^{2}} ; \quad a=d_{n e, n s} \times \cos \left(\varepsilon_{2}^{\prime}\right) \sin (\phi) .
$$

As ISD is a function of $d_{n e, n s}$, we propose an optimization problem for minimizing the ISD as follows.

$$
\underset{d_{n e, n s}}{\operatorname{minimize}} \operatorname{ISD}\left(\varepsilon_{2}, \varepsilon_{2}^{\prime}, \phi, d_{n e, n s}\right)
$$

subject to C.1, C.2, and C.3 in Eq. (5)

By replacing $S N R$ with $S N R_{\text {min }}$ in Eq. (4), and from Eq. (6), the conditions $C .1$ and $C .2$ in Eq. (12) give us the following restriction on the distance between the NGSO satellite and earth terminal systems.

$$
d_{n e, n s} \leq \sqrt{\frac{I_{t h}}{S N R_{\min }}} \times\left(\frac{F\left(d_{g e, n s}, \theta_{s n}, \theta_{g e}\right)}{C}\right) .
$$

By assuming the off-axis angles $\left(\theta_{s n}, \theta_{g e}\right)$, and $d_{g e, n s}$ are not changing, $F$ can be considered as a constant term. The constant term $C$ contains antenna gains 
Table 1: System Parameters

\begin{tabular}{|c|c|}
\hline Parameter & Value \\
\hline Downlink frequency channel & $18.8 \mathrm{GHz}$ \\
\hline$B W$ & $125 \mathrm{MHz}$ \\
\hline$G r x_{e n}(\theta), G r x_{e g}(\theta)$ & ITU-R S.1428 \\
\hline$G t x_{s n}(\theta), G t x_{s g}(\theta)$ & $7 \mathrm{~m}, 12 \mathrm{~m}$ \\
\hline$h_{1}^{\prime}, h_{2}^{\prime}$ & $0.9 \mathrm{~m}$ \\
\hline GSO earth antenna diameter & $1.2 \mathrm{~m}$ \\
\hline NGSO earth antenna diameter & $0.361 \mathrm{~m}$ \\
\hline NGSO satellite antenna diameter & $275 \mathrm{~K}$ \\
\hline$T_{n e}, T_{g e}$ & {$\left[7.79^{\circ} S, 24.25^{\circ} E\right]$} \\
\hline GSO (Eutelsat) satellite [lat,lon,alt $]$ & {$\left[0^{\circ} S \pm 0.1^{\circ}, 9^{\circ} E \pm 0.1^{\circ}, 35,794 \mathrm{~km}\right]$} \\
\hline NGSO (O3b) satellite [lat,lon,alt] & {$\left[1.55^{\circ} S, 31.1^{\circ} E, 8,062 \mathrm{~km}\right]$} \\
\hline GSO terminal [lat,lon] & $10 \mathrm{dBW}$ \\
\hline $\begin{array}{c}\text { NGSO satellite EIRP in the } \\
\text { direction of the NGSO } \\
\text { receiver earth station }\end{array}$ & $-10 \mathrm{~dB}$ \\
\hline$I_{t h}$ & $10 \mathrm{~dB}, 15 \mathrm{~dB}$ \\
\hline$S N R_{\min }$ & \\
\hline
\end{tabular}

of NGSO systems on boresight angle $(\theta=0)$. Therefore, by substituting $d_{n e, n s}$ from Eq. (13) into Eq. (11), the minimum value for ISD can be computed. In next section, we provide results for the cognitive and range-based mechanisms in the co-existence of GSO-NGSO FSS systems. Besides, we provide the results in terms of the link (SNR) outage probability for the NGSO link. We also compare the results for $I S D_{\text {min }}$, using the analytical model in Eq. (11), and the simulation results.

\section{Numerical and Simulation results}

In this section, first, we compare results of the range-based power control with the cognitive range-based power control method. Then, we explain a Monte Carlo technique, which is conducted for computing the $I S D_{\min }$ in our simulation, and we compare this result with the analytical model provided in Section 4 . We have assumed that the traffic statistics do not vary with geographic location or service type. Throughout our numerical results, it is assumed that the NGSO earth terminal is in association with the constellation, and tracks the corresponding NGSO space station once a communication link is established. We increased the range, $d_{n e, n s}$, by moving the NGSO satellite on its longitude. The range between NGSO satellite and earth systems is in associate with the elevation angle on the NGSO earth terminal, $\varepsilon_{2}^{\prime}$. Once the elevation angle of the NGSO earth terminal is lower than the minimum elevation angle, the NGSO earth terminal communicates with the next available NGSO satellite with highest elevation angle $\left(90^{\circ}\right)$. The minimum elevation angle is considered to be $5^{\circ}$ for NGSO earth terminal. Figure 3(a) shows the transmission power of the NGSO satellite operating with the range-based algorithm, in comparison with the NGSO satellite that does not utilize any power control mechanism. As it is depicted in Fig. 3(a), the NGSO satellite without any dynamic power control, operates with the maximum trans- 


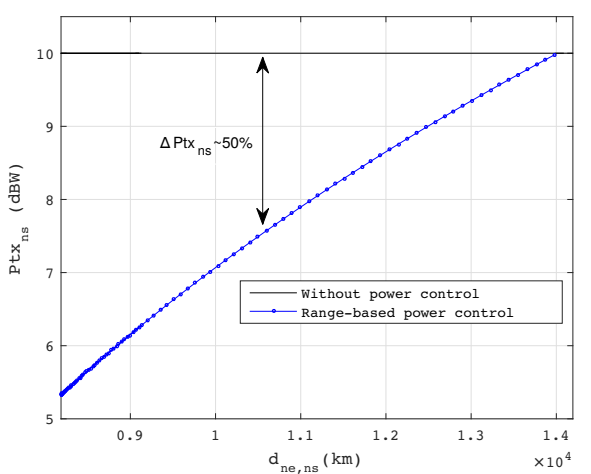

(a)

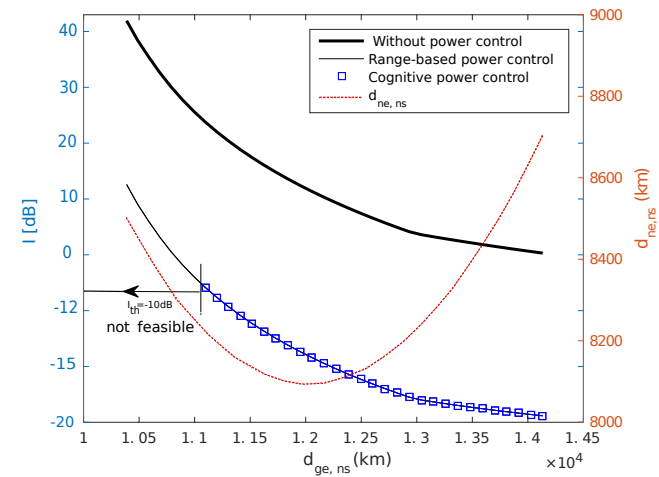

(b)

Fig. 3: a) Comparison of the power transmission on the NGSO satellite, b) Interference level comparison at GSO earth terminal; $S N R_{\text {min }}=15 \mathrm{~dB}, I_{t h}=-10 \mathrm{~dB}, E I R P=$ $10 d B W$.

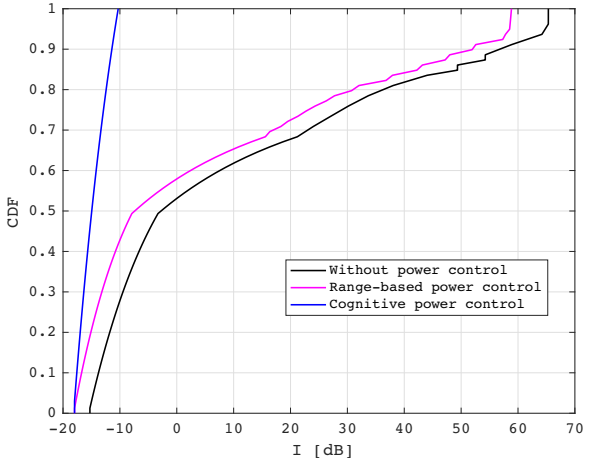

(a)

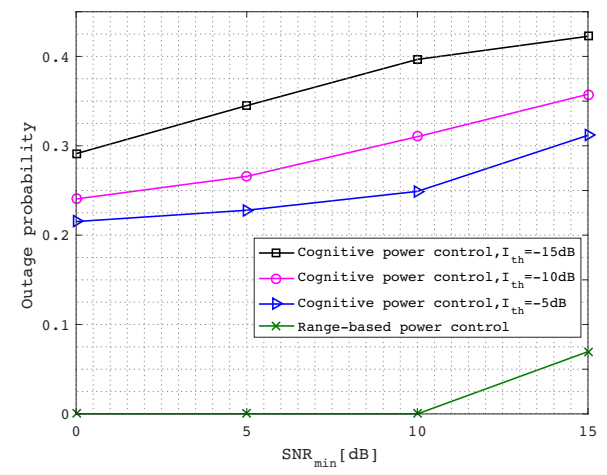

(b)

Fig. 4: a) CDF of interference for GSO earth terminal, $S N R_{\min }=10 \mathrm{~dB}$, b) Probability of outage in SNR for NGSO earth terminal from single NGSO satellite, EIRP = $10 d B W$.

mission power constantly ${ }^{3}$. Wherein, with the range-based power control, the transmission power of the NGSO satellite gradually increases with respect to the distance range, $d_{n e, n s}$. To evaluate the effect of the cognitive range-based power control on the interference level of the GSO earth terminal, please see Fig. 3(b). As it comes from the Fig. 3(b), interference level decreases when the range between NGSO satellite station and GSO earth terminal, $d_{g e, n s}$, increases.

\footnotetext{
${ }^{3}$ Note that in our numerical calculation, we have used the effective isotropically radiated power (EIRP) of the NGSO satellite, which is calculated as $\operatorname{EIRP}[d B W]=$ $10 \times\left(\log _{10}\right.$ Ptx $x_{\max }[W]+\log _{10}$ Gtx $\left.x_{s n}(0)\right)$.
} 
However, the range-based power control has no limit on the interference level of the GSO earth terminal, and it can reach above the $I_{t h}$ with smaller $d_{g e, n s}$. Whereas, with the cognitive range-based power control, the GSO doesn't receive harmful in-line interference above the threshold level. As it is depicted in Fig. 3(b), Eq. (5) has no-feasible solution when the NGSO satellite is getting closer to the GSO earth terminal and getting far from the NGSO earth terminal. Cumulative distribution function (CDF) of the interference level at the GSO earth terminal with respect to the same distances in Fig. 3(b), is shown in Fig. 4(a). To evaluate the effect of the no-feasible solution results on the SNR level of the NGSO earth terminal, please see Fig. 4(b). Using simulation results we have compared the probability of outage in SNR for NGSO earth terminal in this figure. The probability of outage in SNR is calculated as the ratio of the total number of no-feasible solutions and the number of the NGSO satellite movement in the arc between $\left[5^{\circ} \leq \epsilon_{2}^{\prime} \leq 90^{\circ}\right.$. As it comes from the Fig. 4(b), the outage probability on NGSO earth terminal increases for smaller $I_{t h}$ requirements, whereas, in the range-based power control method the outage probability does not vary with $I_{t h}$.

\subsection{Monte Carlo method for choosing $I S D_{\min }$ between earth terminals and their associated GSO-NGSO satellite coverage}

In this method, for N number of times, we simulate a GSO earth terminal location randomly distributed with an associated GSO satellite, which is at a fixed location. The GSO earth terminal locations are computed by choosing a random latitude from $-45^{\circ}$ to $45^{\circ}$ and a random longitude within $0^{\circ}$ to $45^{\circ}$, which is the coverage of the earth from an O3b satellite, that can be interfered with the GSO satellite coverage. The longitude of the GSO earth terminal is chosen by a uniform probability distribution within $\left[0^{\circ}, 45^{\circ}\right]$. Whereas, to take care of the spherical shape of the earth, the latitude distribution of the GSO earth terminal is chosen from following function

$$
F\left(x, 45^{\circ}\right)=(180 / \pi) \arcsin (\sin (45 \times \pi / 180)(2 x-1)),
$$

where $x$ is an uniformly distributed variable between $[0,1]$. Once the GSO earth terminal location is chosen, it is tested to see if the elevation angle, $\varepsilon_{1}$, is within the minimum operating elevation angle of the GSO network or not. The minimum elevation angle at the GSO earth terminal location should be greater than or equal to $10^{\circ}$ (ITU-R S.1325 3). If not, this location is not included as one of the locations simulated. To find the minimum distance between the GSO and NGSO earth terminals, we locate the associated NGSO satellite in random longitudes such that it has minimum elevation angle to the NGSO earth terminal. The minimum elevation angle defines the worst case scenario, where $d_{n e, n s}$ is at the maximum distance. Once the GSO earth terminal location is selected, then, with respect to azimuth angle of the GSO terminal, $0^{\circ} \leq \phi \leq 360^{\circ}$, NGSO earth terminal will be localized. For each azimuth angle, the $I S D_{\min }$ will be found such that its elevation angle with the associated NGSO satellite is $5^{\circ}$, and the 


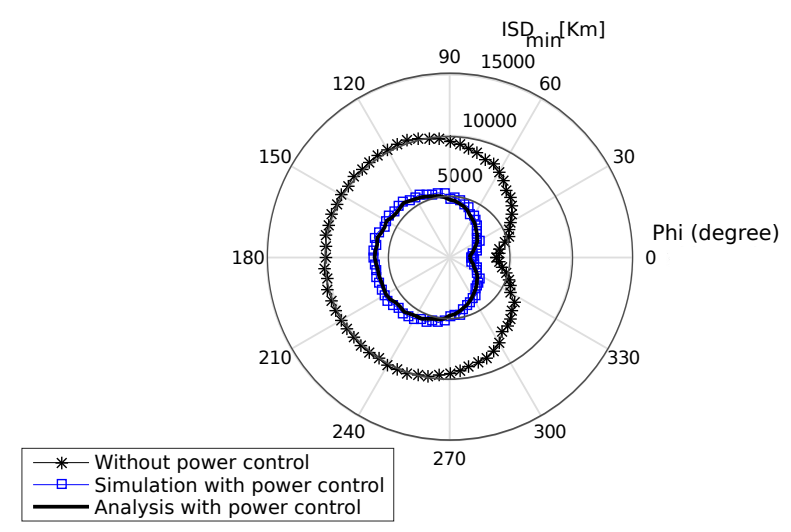

Fig. 5: $I S D_{\text {min }}$ between GSO and NGSO earth terminals.

optimization problem in Eq. (12) is satisfied. We have assumed that the computed $I S D_{\min }$ in this worst case scenario, in which $\varepsilon_{2}^{\prime}=5^{\circ}$, is always greater than the minimum required distance between the two earth terminals. We have compared the result of this simulation with the results of the analysis formula for ISD, using Eq. (11) and (13) in Fig. 5. This result is compared with when the NGSO satellite operates with the maximum transmission power in Fig. 5. As it comes from this figure, the cognitive range-based power control method can significantly reduce the $I S D_{\min }$ between FSS earth terminals.

\section{Conclusions}

In this work, we proposed to employ a cognitive range-based power control mechanism for an NGSO satellite operating in the downlink mode. We have examined the proposed power control method with the range-based power control mechanism suggested by ITU-R S.1325 3. The results verify that the cognitive power control method avoids the harmful in-line interference on the victim receiver. We note that although finding the distance from the satellite to an adjacent earth terminal is not practically possible, still our optimization problem can benefit the victim receiver by maintaining $I_{t h}$ as a constant all the time. This threshold value lessens the imposed harmful interference on the adjacent earth terminals. We also proposed an optimization problem for finding the minimum inter-side distance between the FSS earth stations. The proposed ISD optimization technique can play a critical role in optimizing the network planing strategies.

\section{Acknowledgement}

This work was supported by the National Research Fund, Luxembourg under the project "HSBNet".

\section{References}

1. Evans, B., Werner, M., Lutz, E., Bousquet, M., Corazza, G.E., Maral, G.: Integration of satellite and terrestrial systems in future multimedia communications. Wireless Communications, IEEE, 12, 5, 72-80 (2005) 
2. Mohammed, H., Jakllari, G., Paillassa, B.: Network pruning for extending satellite service life in LEO satellite constellations. Wireless Networks, 1-13 (2015)

3. Kolodzy, P.: Interference Avoidance Spectrum policy task force. Technical report, Federal Commun, 02-135 (2002)

4. Laurent, C., Alamaac, A., Bousquet, M.: Interference and fade mitigation techniques for $\mathrm{Ka}$ and $\mathrm{Q} / \mathrm{V}$ band satellite communication systems. Proc. 2nd Int. Workshop of COST Action, 280 (2008)

5. Dae-Sub, O., Ku, B.J., Kim, S.: Compatibility study on terrestrial radio system operated in the coverage of multi-beam satellite system. Communications (APCC), 17th Asia-Pacific Conf. on. IEEE (2011)

6. Koletta, M., Milas, V.: Determination of the coordination area for mobile earth stations operating with geostationary space stations in the frequency band shared with the terrestrial services. IEEE VTC, 2707-2710 (2005)

7. Chatzinotas, S., Ottersten, B., DeGaudenzi, R.: Cooperative and Cognitive Satellite Systems. Academic Press (2015)

8. Sharma S.K., et al.: Satellite cognitive communications: Interference modelling and techniques selection. ASMS Conf. and 12th SPSC workshop, 111-118 (2012)

9. Chatzinotas, S., Sharma, S.K., Ottersten, B.: Frequency packing for interference alignment-based cognitive dual satellite systems. Vehicular Technology Conference (VTC Fall), IEEE, 1-7 (2013)

10. Sharma, S.K., Chatzinotas, S., Ottersten, B.: In-line interference mitigation techniques for spectral coexistence of GEO and NGEO satellites. International Journal of Satellite Communications and Networking, 34, 1, 11-39 (2016)

11. Wang, A.W.: Method and apparatus for providing wideband services using medium and low earth orbit satellites. U.S. Patent No. 7,627,284 (2009)

12. Vatalaro, F., Corazza, G., Caini, C., Ferrarelli, C.: Analysis of LEO, MEO, and GEO global mobile satellite systems in the presence of interference and fading. IEEE Journal on selected areas in communications, 13.2, 291-300 (1995)

13. Reed, A., Posen, M.: Interference in the fixed satellite service bands between the feeder-links of networks using nongeostationary satellites and network using geostationary satellites. 3rd European Conf. Satellite Commun. ECSC-3: Manchester, UK, 251-256 (1993)

14. Sharma, S.K., Chatzinotas, S., Ottersten, B.: Transmit beamforming for spectral coexistence of satellite and terrestrial networks. 8th Int. conf. CROWNCOM, 275-281 (2013)

15. Lei, S., Sung, K.W., Zander, J.: Controlling aggregate interference under adjacent channel interference constraint in TV white space. 7th Int. conf. CROWNCOM, 1-6. IEEE (2012)

16. Farrokhi, R., Liu, K.J., Tassiulas, L.: Transmit beamforming and power control for cellular wireless systems. Selected Areas in Communications, IEEE Journal, 16.8, 1437-1450, (1998)

17. Alvin, F., Eytan, M., Tsitsiklis, J.N.: Optimal energy allocation and admission control for communications satellites. Networking, ACM Transactions on, 11, 3, 488500, IEEE (2003)

18. Ganho, Francisco, et al.: Energy-efficient QOS provisioning in demand assigned satellite NDMA schemes. 21st Int. Conf. ICCCN, 1-8, IEEE (2012)

19. Dae-Sub, O., et al.: A study on the separation distance for frequency sharing between GSO network and terrestrial network in Ka band. Vehicular Technology Conference, VTC Spring, 2967-2971, IEEE (2008) 